\title{
Orange Juice and Its Component, Hesperidin, Decrease the Expression of Multidrug Resistance-Associated Protein 2 in Rat Small Intestine and Liver
}

\author{
Minoru Watanabe, ${ }^{1,2}$ Naoki Matsumoto, ${ }^{1}$ Yuko Takeba, ${ }^{1}$ Toshio Kumai, ${ }^{1,3}$ \\ Masami Tanaka, ${ }^{1,2}$ Shinobu Tatsunami, ${ }^{4}$ Sachiko Takenoshita-Nakaya, ${ }^{1}$ \\ Yoshie Harimoto, ${ }^{1}$ Yuichi Kinoshita, ${ }^{1}$ and Shinichi Kobayashi ${ }^{1}$ \\ ${ }^{1}$ Department of Pharmacology, St. Marianna University School of Medicine, 2-16-1 Sugao, Miyamae-Ku, \\ Kawasaki, Kanagawa 216-8511, Japan \\ ${ }^{2}$ Institution for Animal Experimentation, St. Marianna University Graduate School of Medicine, Kawasaki, \\ Kanagawa 216-8511, Japan \\ ${ }^{3}$ Department of Pharmacogenomics, St. Marianna University Graduate School of Medicine, Kawasaki, \\ Kanagawa 216-8511, Japan \\ ${ }^{4}$ Unit of Medical Statistics, Faculty of Medical Education and Culture, St. Marianna University School of Medicine, \\ Kawasaki, Kanagawa 216-8511, Japan \\ Correspondence should be addressed to Minoru Watanabe,m4wata@marianna-u.ac.jp \\ Received 13 September 2010; Revised 25 February 2011; Accepted 24 March 2011 \\ Academic Editor: Phillip E. Klebba
}

Copyright (C) 2011 Minoru Watanabe et al. This is an open access article distributed under the Creative Commons Attribution License, which permits unrestricted use, distribution, and reproduction in any medium, provided the original work is properly cited.

We investigated the effects of orange juice (OJ) or hesperidin, a component of OJ, on the pharmacokinetics of pravastatin (PRV) and the expression of both protein and mRNA of multidrug resistance-associated protein 2 (Mrp2) in the rat small intestine and liver. Eight-week-old male Sprague-Dawley rats were used in this study. OJ or a $0.079 \%$ hesperidin suspension was administered orally for 2 days. Tap water was given as a control. A single dose of PRV at $100 \mathrm{mg} / \mathrm{kg}$ p.o. was administered after 2 days of OJ, hesperidin, or tap water ingestion. The AUC, $C_{\max }$, and $t_{1 / 2}$ values of PRV were significantly increased in OJ group. Mrp2 protein and mRNA levels in the small intestine and liver, respectively, were significantly decreased after the ingestion of OJ. The same results were obtained with hesperidin. These results suggest that the changes in PRV pharmacokinetic parameters and the decrease in Mrp2 expression caused by $\mathrm{OJ}$ are due to hesperidin in the juice.

\section{Introduction}

Many reports have confirmed that grapefruit juice (GFJ) alters the bioavailability of lipophilic drugs due to its inhibitory effect on CYP3A4 or on the efflux transporter Pglycoprotein $[1,2]$. However, there are few reports on the effects of other types of juice on the pharmacokinetics of hydrophilic drugs.

Pravastatin (PRV) is an HMG-CoA reductase inhibitor administered as a sodium salt of the active hydroxy acid form. The bioavailability of PRV with oral administration is about $20 \%$ of the total dose [3]. Dresser and Bailey reported that orange juice $(\mathrm{OJ})$ affects the pharmacokinetics of fexofenadine [2]. However, the role of OJ in the pharmacokinetics of hydrophilic drugs was unclear. Therefore, we investigated the effects of OJ (short-term ingestion) on PRV pharmacokinetics and found that ingesting $\mathrm{OJ}$ increased the area under the plasma drug concentration-time curve (AUC) and peak plasma concentration $\left(C_{\max }\right)$ of PRV in rats [4]. On the other hand, it is known that multidrug resistanceassociated protein 2 (MRP2) is involved in the transport of PRV $[5,6]$. Thus, we also investigated the effects of shortterm $\mathrm{OJ}$ ingestion on the level of Mrp2 protein and mRNA in the small intestine and liver. However, there was no change 
in Mrp2 levels after short-term OJ ingestion. Lilja et al. [7] reported a study in which volunteers drank $200 \mathrm{~mL}$ doublestrength juice three times daily for 2 days. Dresser and Bailey [2] conducted a study in which the volume and schedule of OJ administration were $300 \mathrm{~mL}$ with 2 drug tablets, followed by $150 \mathrm{~mL}$ every 0.5 to $3.0 \mathrm{~h}$ (total volume, $1.2 \mathrm{~L}$ ). Based on those reports, we selected the dose and dosage schedule of $\mathrm{OJ}$ in the current study (2-days OJ ingestion). Since a decrease in Mrp2 expression after 2-day OJ ingestion was observed, we reexamined the effects of 2 day of OJ on PRV pharmacokinetics.

The predominant flavanone in the serum after OJ ingestion is hesperetin $[8,9]$. This flavanone is common in plants, where they are conjugated to glycosides. Hesperetin is present in oranges as hesperetin-7-O-rutinoside (hesperidin). Hesperidin is metabolized to hesperetin in the small intestine and assumes its active form. We also investigated the influence of hesperidin on the pharmacokinetics of PRV and the expression of Mrp2 in the rat small intestine and liver.

\section{Materials and Methods}

2.1. OJ Study I. Eight-week-old male Sprague-Dawley rats were used in this study. OJ was administered orally every hour for a total of 8 times on the 1st day and 5 times on the 2nd day. The initial dose was $5 \mathrm{~mL} / \mathrm{kg}$, and each consecutive dose was $2.5 \mathrm{~mL} / \mathrm{kg}$. Tap water was given to the control group. A single dose of PRV at $100 \mathrm{mg} / \mathrm{kg}$ p.o. was administered $1 \mathrm{~h}$ after the last ingestion of OJ or tap water. Serial blood samples were collected intravenously from the tail vein. Venous blood samples were placed in tubes containing $5 \mu \mathrm{L}$ of $0.2 \mathrm{M}$ ethylenediaminetetraacetic acid (EDTA) 15, 30, $45,60,75,90,120,150$, and $180 \mathrm{~min}$ after the administration of PRV to measure plasma PRV concentrations. Blood samples were immediately centrifuged at $2000 \mathrm{~g}$ for $10 \mathrm{~min}$ at $4^{\circ} \mathrm{C}$ for plasma collection and stored at $-20^{\circ} \mathrm{C}$ until analysis.

PRV levels were measured according to the methods of Otter and Mignat [10] and Lacona et al. [11]. The internal standard solution was prepared by dissolving triamcinolone acetonide (Sigma Chemical, St. Louis, MO, USA) in methanol and a $300 \mu \mathrm{L}$ aliquot containing $1 \mu \mathrm{g} / \mathrm{mL}$ of this internal standard solution was added to $100 \mu \mathrm{L}$ of plasma. Then, $900 \mu \mathrm{L}$ of water was added and mixed. The samples were loaded on a $\mathrm{C}_{18}$ preparatory solid-phase extraction column (Sep-Pak cartridge, Waters Co., Milford, MA, USA) conditioned with $2 \mathrm{~mL}$ of methanol and $3 \mathrm{~mL}$ of water. The cartridges were then washed with $2 \mathrm{~mL}$ of $80 \%$ methanol and allowed to dry for $60 \mathrm{~min}$ under air. After evaporation, the residue was dissolved in $500 \mu \mathrm{L}$ of methanol and allowed to dry for an additional $15 \mathrm{~min}$. Finally, the samples were eluted with $300 \mu \mathrm{L}$ of methanol and $100 \mu \mathrm{L}$ was injected on the HPLC column. The chromatographic system consisted of a DP-8020 pump, an AS-8020 autosampler, and a UV detector (UV-8020, Tosoh Co., Tokyo, Japan). Separation was achieved on a Cosmosil 5C18-AR column ( $5 \mu \mathrm{m}$ particlesize, $4.6 \times 150 \mathrm{~nm}$ ID, Nakalai Tesque, Kyoto, Japan). The chromatographic data were collected and processed with an LC-8020 multistation system (Tosoh Co.). The mobile phase was $74 \%$ ammonium phosphate $(0.05 \mathrm{M}, \mathrm{pH} 3.5)$ and $26 \%$ acetonitrile. The flow rate was $1.0 \mathrm{~mL} / \mathrm{min}$ with column temperatures maintained at $37^{\circ} \mathrm{C}$. The UV detector was set at $239 \mathrm{~nm}$. Under these conditions, the retention times for PRV and the internal standard were 13 and 23 min, respectively. The standard curve of PRV was linear over the range tested $\left(10 \mathrm{pg} / \mathrm{mL}-500 \mathrm{ng} / \mathrm{mL}\right.$; slope 4.156 , intercept $-1.117, R^{2}=$ 0.999 [HPLC peak area versus PRV content]). The interassay and intra-assay coefficients of variation were within $5.12 \%$ and $1.42 \%$, respectively.

Pharmacokinetic parameters for PRV were obtained from plasma concentration-time profiles. AUC $(0-3.0 \mathrm{~h})$ was determined using the trapezoidal rule. The $t_{1 / 2}$ was calculated as $=\operatorname{Ln}(2 / \beta)$, where $\beta$ is the slope of the terminal elimination phase obtained by least-squares linear regression. The maximum plasma concentration $\left(C_{\max }\right)$ and time to $C_{\max }\left(T_{\max }\right)$ were determined directly from the observed data. The value of $\mathrm{CL} / \mathrm{f}$ was estimated using a nonlinear regression program [12].

All experiments were conducted in accordance with the Guidelines for Animal Experimentation, St. Marianna University School of Medicine and had the approval of the Animal Research Committee, Institute for Animal Experimentation, St. Marianna University Graduate School of Medicine.

2.2. OJ Study II. OJ and tap water was given to 8-weekold male Sprague-Dawley rats on the same schedule and at the same volume as in OJ study I. The small intestine and liver were removed $1 \mathrm{~h}$ after the last ingestion of OJ or tap water, and plasma membrane fractions were prepared [13]. In brief, the small intestine and liver were homogenized in $100 \mathrm{mM}$ Tris- $\mathrm{HCl}, \mathrm{pH} 7.5$, containing a protease inhibitor mixture (Roche Diagnostics, Mannheim, Germany). The homogenate was centrifuged at $1500 \mathrm{~g}$ for $15 \mathrm{~min}$, and the supernatant was then centrifuged at $100,000 \mathrm{~g}$ for $35 \mathrm{~min}$. The resulting pellets were washed, resuspended in Tris $\mathrm{HCl}$ buffer, and stored at $-80^{\circ} \mathrm{C}$ prior to analysis.

Mrp2 protein levels were analyzed using Western blotting. Protein concentrations were determined using the BioRad Protein Assay (Bio-Rad, Hercules, CA, USA). Protein samples $(50 \mu \mathrm{g}$ each) were subjected to SDS-PAGE on $8 \%$ SDS polyacrylamide gel. The proteins were transferred to a nitrocellulose membrane (Hybond-ECL, Amersham Bioscience, Piscataway, NJ, USA), and the membrane was blocked with Tris-buffered saline (TBS)-0.1\% Tween-20 containing 5\% skim milk. The membrane was then incubated for $1 \mathrm{~h}$ with anti-MRP2 antibody (abcam, Cambridge, UK) and anti-MRP2 antibody (Chemicon) diluted $1: 100$ in TBS. After three washes with $0.1 \%$ Tween-20 in TBS (TTBS), the membrane was incubated for $1 \mathrm{~h}$ with peroxidelabeled antimouse IgG antibody (Cappel, Aurora, OH, USA) diluted 1:2500 in T-TBS and developed with the electrochemiluminescence (ECL) method (ECL plus Western blotting detection system, Amersham Bioscience). The intensity of the staining of each membrane was measured with a densitometer (Densitograph, ATTO Co., Tokyo, Japan). The intensity of bands was then measured. 
Levels of Mrp2 mRNA were determined with real-time PCR (LightCycler, Roche Diagnostics) as described previously, with slight modification [14]. Samples of liver and intestinal tissue were then collected in the same manner as for Western blot analysis. Total RNA was extracted from the intestinal tissue with an RNA extraction kit (RNAgents Total RNA Isolation System, Promega Co., Madison, WI, USA). Two micrograms of total RNA were reversed transcribed with $100 \mathrm{U}$ of Moloney murine leukemia virus reverse transcriptase (RETROscript kit, Ambion Inc., Austin, TX, USA) in $20 \mu \mathrm{L}$ of total reaction volume containing reversetranscriptase buffer, random primer, dNTP, and RNase inhibitor. PCR was performed in $20 \mu \mathrm{L}$ of total reaction volume containing $2 \mu \mathrm{g}$ of $\mathrm{cDNA}$, primers specific for MRP2 and glyceraldehyde-3-aldehydro dehydrogenase (GAPDH), $\mathrm{MgCl}_{2} 3 \mathrm{mmoL} / \mathrm{L}$, and LightCycler FastStart DNA Master SYBR Green I (Roche Diagnostics). The cycling protocol consisted of one cycle of $10 \mathrm{~min}$ at $95^{\circ} \mathrm{C}$ followed by 40 cycles of denaturation for $15 \mathrm{~s}$ at $95^{\circ} \mathrm{C}$, annealing for $10 \mathrm{~s}$ at $58^{\circ} \mathrm{C}$, and extension for $21 \mathrm{~s}$ at $72^{\circ} \mathrm{C}$. The primers for MRP2 were $5^{\prime}$-TTAACCGGGGACGTTG-3' (sense) and $5^{\prime}$-GCCGATACCGCACTTGA- $3^{\prime}$ (antisense) and those for GAPDH were $5^{\prime}$-CTGAGTATGTCGTGGAGTCTA- ${ }^{\prime}$ (sense) and $5^{\prime}$-CTGCTTCACCACCTTCTTGAT-3' (antisense). Serial dilutions of the standard cDNA were also used for parallel amplifications. The threshold cycles $\left(C_{t}\right)$ were calculated with LightCycler software (ver. 5.32). Standard curves were plotted with $C_{t}$-versus-log cDNA quantities, and the quantities of samples were determined from the standard curves. In addition, Mrp2 mRNA levels were normalized to those of GAPDH in each sample.

2.3. Hesperidin Study. Hesperidin instead of OJ was given to 8-week-old male Sprague-Dawley rats using the same schedule and volume as for the OJ studies. Tap water was also given to the rats as a control. The hesperidin suspension was prepared at a concentration of $79 \mathrm{mg}$ per $100 \mathrm{~mL}$ of tap water $(0.079 \%)$. This concentration was equal to that contained in OJ [15]. The pharmacokinetic study of PRV and measurement of Mrp2 protein and mRNA were performed using the same methods as in OJ studies.

2.4. Statistical Analysis. Results are expressed as mean \pm $\mathrm{SD}$. Mean values were compared with one-way analysis of variance followed by the Scheffe test. $P$ values of less than .05 were considered statistically significant.

\section{Results}

3.1. OJ Study I. Plasma PRV concentration-time profiles are shown in Figure 1. After the administration of PRV, plasma concentrations increased in a time-dependent manner and subsequently decreased gradually. Significant increases in the AUC $(141 \%, P<.05), C_{\max }(134 \%, P<.05)$, and $t_{1 / 2}(184 \%$, $P<.05)$ values of PRV were seen in the OJ group compared with the control group, although CL/f value was significantly decreased after $\mathrm{OJ}$ ingestion and the $t_{\max }$ values did not differ significantly (Table 1).
TABLE 1: Effect of orange juice on the pharmacokinetic parameters of single-orally administrated pravastatin on rats.

\begin{tabular}{lccc}
\hline Parameter & $\begin{array}{c}\text { Control } \\
(n=6)\end{array}$ & $\begin{array}{c}\text { Orange Juice } \\
(n=6)\end{array}$ & $\begin{array}{c}\text { Ratio } \\
(\mathrm{OJ} / \text { Cont })\end{array}$ \\
\hline$C_{\max }(\mathrm{ng} / \mathrm{mL})$ & $120.8 \pm 17.2$ & $161.4 \pm 26.4^{*}$ & 1.34 \\
$T_{\max }(\mathrm{min})$ & $48.0 \pm 12.5$ & $50.0 \pm 12.2$ & $\mathbf{1 . 0 4}$ \\
$t_{1 / 2}(\mathrm{~min})$ & $75.5 \pm 18.4$ & $138.8 \pm 58.5^{*}$ & 1.84 \\
$\mathrm{CL} / \mathrm{f}(\mathrm{L} / \mathrm{hr})$ & $105.0 \pm 22.8$ & $49.0 \pm 17.4^{* *}$ & 0.47 \\
AUC $(\mathrm{ng} \cdot \mathrm{hr} / \mathrm{mL})$ & $207.1 \pm 21.4$ & $291.3 \pm 78.0^{*}$ & $\mathbf{1 . 4 1}$ \\
\hline
\end{tabular}

The data are expressed as the mean \pm S.D.

${ }^{*} P<.05$ and ${ }^{* *} P<.01$, significantly different from control.

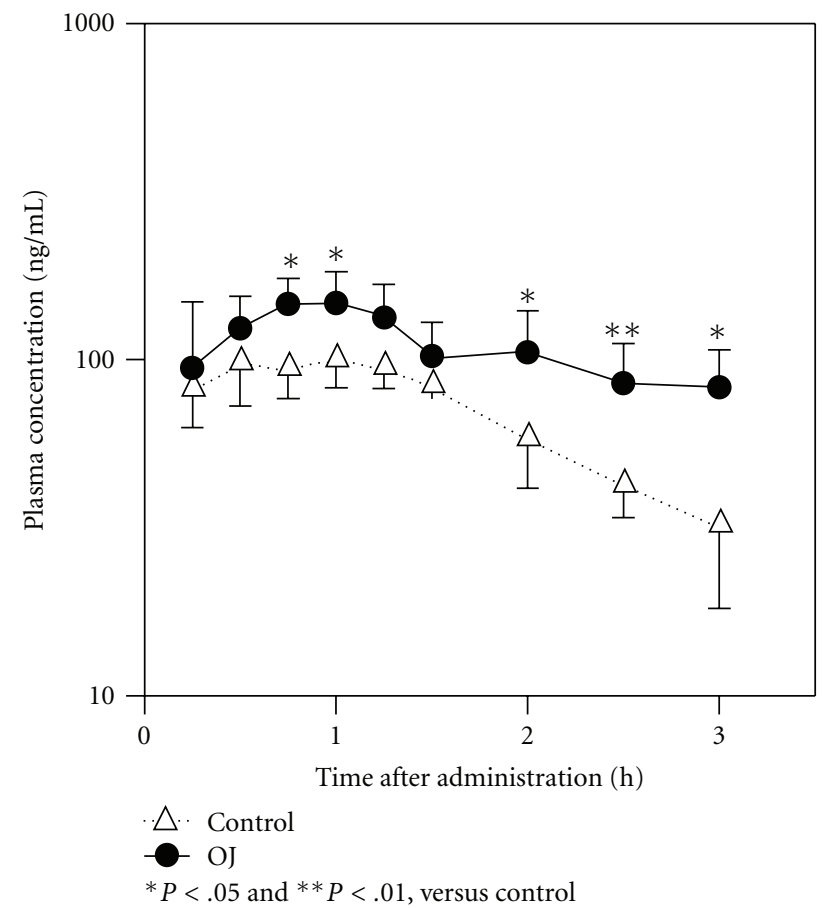

Figure 1: Effect of orange juice (OJ) on the plasma concentration of pravastatin (PRV) in Sprague-Dawley rats. OJ was administered orally 8 times (1st day) and 5 times (2nd day) at hourly intervals. The starting dose of OJ was $5 \mathrm{~mL} / \mathrm{kg}$, and each consecutive dose was $2.5 \mathrm{~mL} / \mathrm{kg}$. Tap water instead of OJ was given to the control group. A single dose of PRV at $100 \mathrm{mg} / \mathrm{kg}$ p.o. was administered $1 \mathrm{~h}$ after the last ingestion of OJ or tap water. PRV levels were measured using the HPLC method described in Section 2.

3.2. OJ Study II. The MRP2 protein level was significantly decreased in the OJ group to $36.7 \%$ in the liver (Figure 2(a)), and to $33.9 \%$ in the intestine (Figure 2(b)) compared with the respective control values. The Level of Mrp2 mRNA was also significantly decreased in the OJ group to $42.0 \%$ in the liver (Figure 3(a)) and to $66.4 \%$ in the intestine (Figure 3(b)) compared with the respective control values.

3.3. Hesperidin Study. Figure 4 shows the effects of hesperidin on plasma PRV concentration-time profiles. Significant increases in the AUC $(147 \%, P<.05)$, the $C_{\max }$ $(138 \%, P<.05)$, and $t_{1 / 2}(203 \%, P<.05)$ values of 


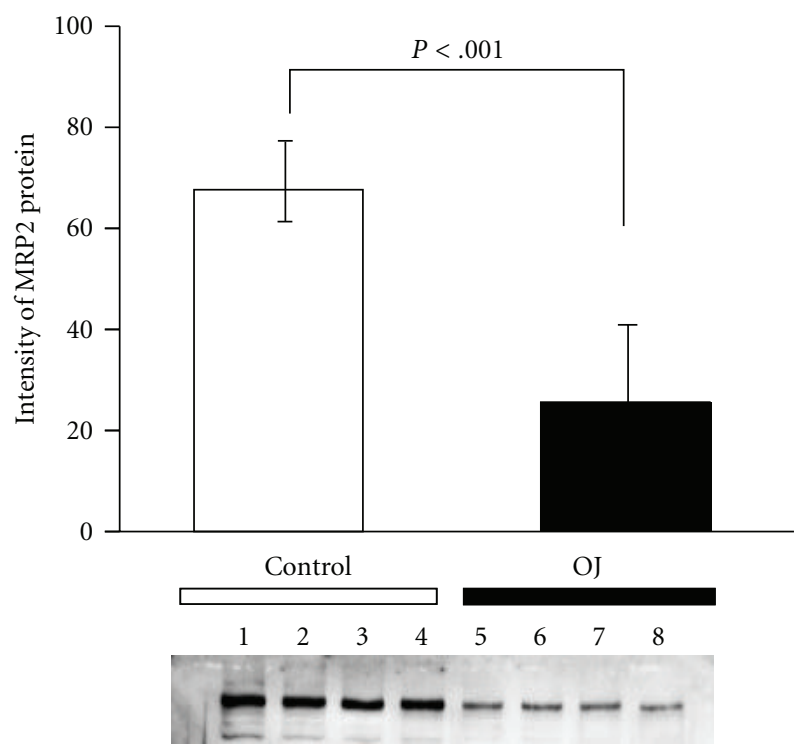

(a) Liver

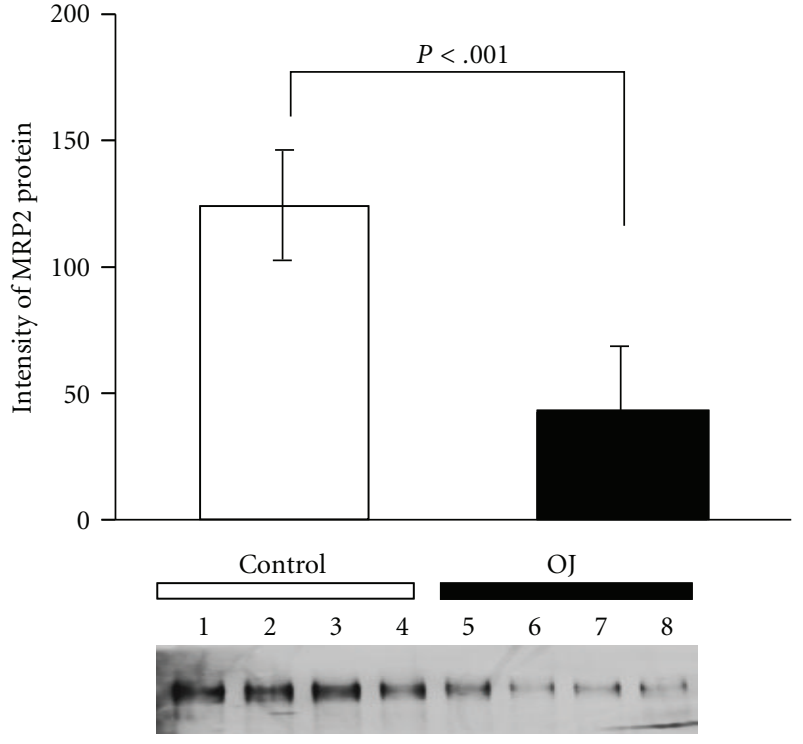

(b) Small intestine

FIGURE 2: Effect of orange juice (OJ) on Mrp2 protein levels in both the liver (a) and small intestine (b) in rats. OJ or tap water was given according to the same schedule as in Figure 1. The liver and small intestine were removed $1 \mathrm{~h}$ after the last ingestion of OJ or tap water, respectively, and plasma membrane fractions were prepared. Details of the Western blot analysis are described in Section 2.

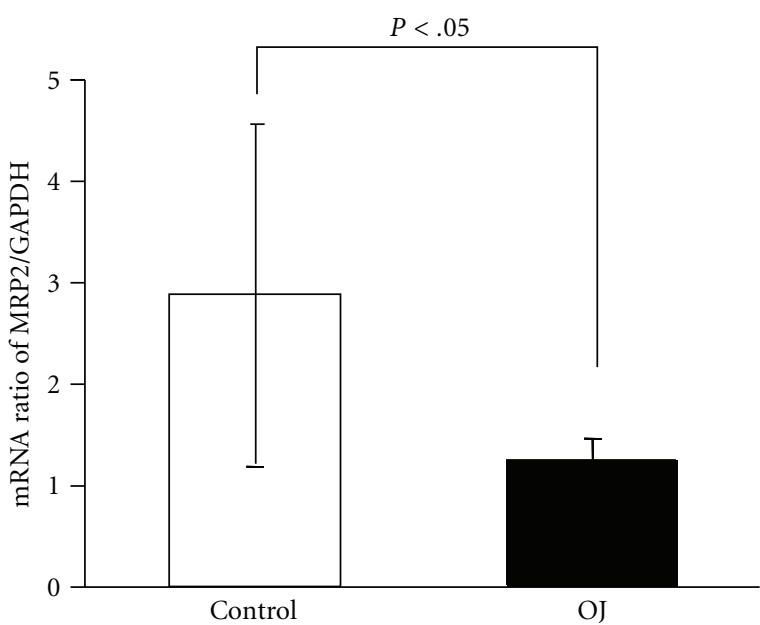

(a) Liver

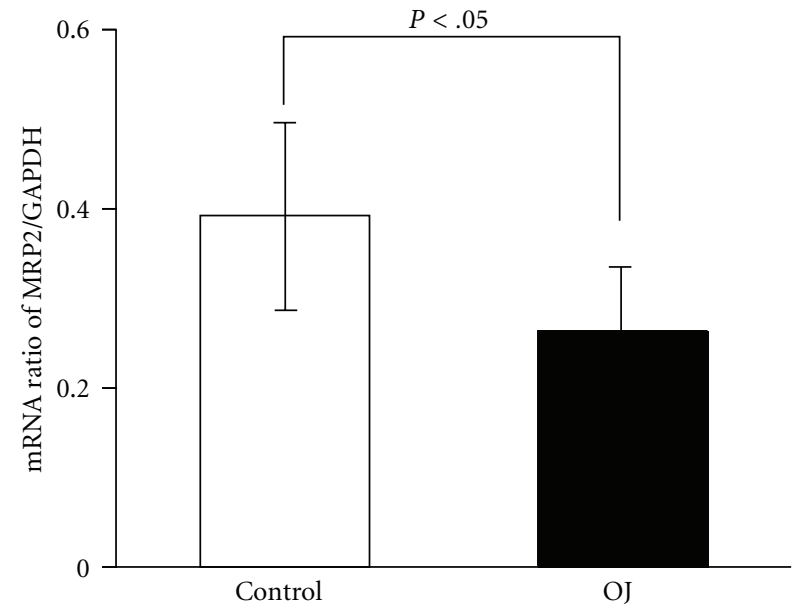

(b) Small intestine

FIGURE 3: Effect of orange juice (OJ) on the expression of Mrp2 mRNA in both the liver (a) and small intestine (b) in rats. Tap water or OJ was given according to the same schedule as in Figure 1. The liver and small intestine were removed $1 \mathrm{~h}$ after the last ingestion of OJ or tap water, respectively, and total RNA was extracted from these tissues directly with the RNAgent Total RNA Isolation System. Levels of Mrp2 mRNA were determined with real-time PCR as described in Section 2.

PRV were observed in the heperidin group compared with the control group, although CL/f value was significantly decreased after hesperidin ingestion and the $t_{\max }$ values did not differ significantly (Table 2).

The Mrp2 protein level was significantly decreased in the hesperidin group to $50.1 \%$ in the liver (Figure 5(a)) and to $28.3 \%$ in the intestine (Figure 5(b)) compared with the respective control values. The level of Mrp2 mRNA was also significantly decreased in the hesperidin group to $53.8 \%$ in the liver (Figure 6(a)) and to $70.4 \%$ in the intestine (Figure 6(b)) of the respective control values.

\section{Discussion}

In the present study, we found significantly increases in the AUC and $C_{\max }$ values and prolonged $t_{1 / 2}$ of PRV in rats in 
TABLE 2: Effect of hesperidin on the pharmacokinetic parameters of single-orally administrated pravastatin on rats.

\begin{tabular}{lccc}
\hline Parameter & $\begin{array}{c}\text { Control } \\
(n=6)\end{array}$ & $\begin{array}{c}\text { Hespridin } \\
(n=6)\end{array}$ & $\begin{array}{c}\text { Ratio } \\
(\text { Hes/Cont })\end{array}$ \\
\hline$C_{\max }(\mathrm{ng} / \mathrm{mL})$ & $116.5 \pm 10.5$ & $160.1 \pm 20.7^{*}$ & 1.38 \\
$T_{\max }(\mathrm{min})$ & $52.5 \pm 8.2$ & $52.5 \pm 12.6$ & 1.00 \\
$t_{1 / 2}(\mathrm{~min})$ & $69.1 \pm 3.7$ & $140.3 \pm 30.8^{*}$ & 2.03 \\
$\mathrm{CL} / \mathrm{f}(\mathrm{L} / \mathrm{hr})$ & $111.2 \pm 44.4$ & $55.9 \pm 37.5^{*}$ & 0.55 \\
$\mathrm{AUC}(\mathrm{ng} \cdot \mathrm{hr} / \mathrm{mL})$ & $213.5 \pm 21.1$ & $319.3 \pm 23.7^{*}$ & 1.47 \\
\hline
\end{tabular}

The data are expressed as the mean \pm S.D.

${ }^{*} P<.05$, significantly different from control.

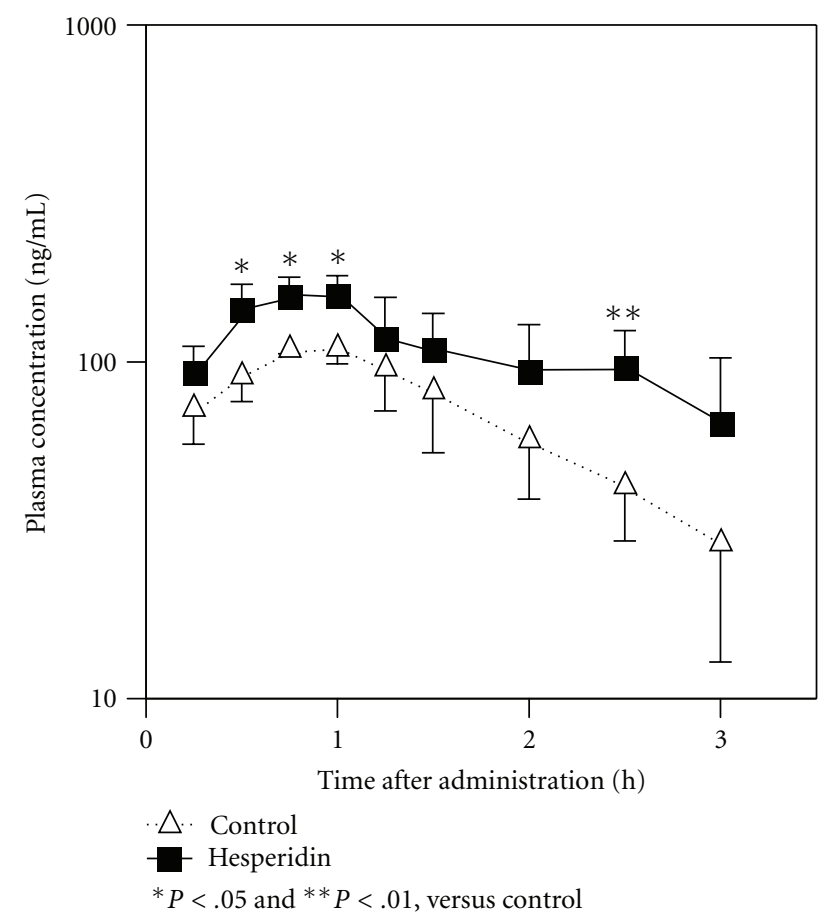

FIGURE 4: Effect of hesperidin (Hes) on the plasma concentration of pravastatin (PRV) in Sprague-Dawley rats. A hesperidin suspension was prepared at a concentration of $79 \mathrm{mg}$ per $100 \mathrm{~mL}$ of tap water $(0.079 \%)$. The suspension instead of OJ was given according to the same schedule and volume as in the OJ study (Figure 1).

the OJ group. These results suggest that $\mathrm{OJ}$ intake increases the rate of absorption of PRV based on the increased $C_{\max }$ and decreases the rate of elimination of PRV from the systemic circulation based on the prolonged $t_{1 / 2}$.

PRV is moved by transporters [16-19], while CYP3A4 plays only a minor role in the biotransformation of PRV [20]. Therefore, oxidative enzymes in the intestine or liver may not be involved in changes in the pharmacokinetics $\left(C_{\max }, \mathrm{AUC}\right.$, and $t_{1 / 2}$ ) of PRV with OJ intake.

Mrp2 participates in the biliary excretion of PRV in the liver $[18,19]$, and the transporter is also present in the small intestine [21-23]. Mrp2 may play the role of an excretion transporter in the small intestine. However, there has been no report on the influence of OJ on Mrp2 protein and its mRNA levels in the liver and small intestine. Here we found that the amount of Mrp2 protein decreased in both the liver and small intestine after the ingestion of OJ. Since Mrp2 mRNA levels also decreased with the ingestion of OJ, it was thought that the loss of Mrp2 protein was a result of decreased gene expression.

Mrp2, an organic anion transporter, excretes xenobiotics from the apical membranes of intestinal epithelial cells into the intestinal lumen [24]. Therefore, the increase in $C_{\max }$ after ingestion of $\mathrm{OJ}$ is thought to be due to the relative increase in PRV absorption in the small intestine, which is based on the decrease in Mrp2 expression in the small intestine caused by OJ.

Eighty percent of PRV is excreted via the liver [25], and Mrp2 participates in this excretion [18, 19]. Therefore, it was also thought that the decrease in hepatic Mrp2 after the ingestion of OJ caused the decrease in the biliary excretion of PRV (including a decrease in first-pass excretion), and this decrease may cause the prolongation of $t_{1 / 2}$ and the increase in the $C_{\max }$ of PRV.

We next investigated whether a specific component in OJ reduces Mrp2 levels in the liver and small intestine. The pharmacokinetic parameters $\left(C_{\max }, \mathrm{AUC}\right.$, and $\left.t_{1 / 2}\right)$ of PRV changed after the ingestion of OJ (in this study) but not after the ingestion of GFJ [7]. These results suggest that a component that is more abundant in OJ than in GFJ reacts with the transporter for PRV.

The predominant flavanone in blood is hesperetin when $\mathrm{OJ}$ is ingested and naringenin when GFJ is ingested $[8,9]$. These flavanones are common in plants where they are conjugated to glycosides. Hesperetin is present in oranges as hesperetin-7-O-rutinoside (hesperidin). For these reasons, we also investigated the influence of hesperidin, instead of hesperetin, on the pharmacokinetic parameters $\left(C_{\max }, \mathrm{AUC}\right.$, and $t_{1 / 2}$ ) of PRV and the expression of Mrp2 protein and mRNA in the rat small intestine and liver.

Hesperidin significantly increased the AUC, $C_{\max }$, and $t_{1 / 2}$ values of PRV. The level of Mrp2 protein and mRNA decreased with the ingestion of hesperidin in the small intestine and liver.

When we compared the effects of $\mathrm{OJ}$ and hesperidin, both increased the bioavailability of PRV in rats. Since the quantity of hesperidin ingested was the same as the quantity in OJ, it was suggested that the decrease in Mrp2 expression in the liver and small intestine after the ingestion of $\mathrm{OJ}$ was caused by the hesperidin in the juice.

In rats, it was reported that oatp2 and oatp3 are also localized at the apical membrane of intestinal epithelial cells [26]. PRV is a substrate for oatp1 and oatp2 in several tissues [27]. The physiologic role of the intestinal OATP family is the transport of drugs from the intestinal tract into the portal circulation. In the liver, PRV is taken up via a mechanism mediated by the OATP family (oatp1, oatp2 [OATP C]) from the portal circulation to hepatocytes [28]. For this reason, we investigated the effects of $\mathrm{OJ}$ or hesperidin (2-day ingestion) on the level of the oatp2 protein in the liver or small intestine. Consequently, although the data are not shown, the oatp2 protein level increased in the small intestine after 2-day OJ ingestion, but the oatp 2 protein level in liver did not change. 


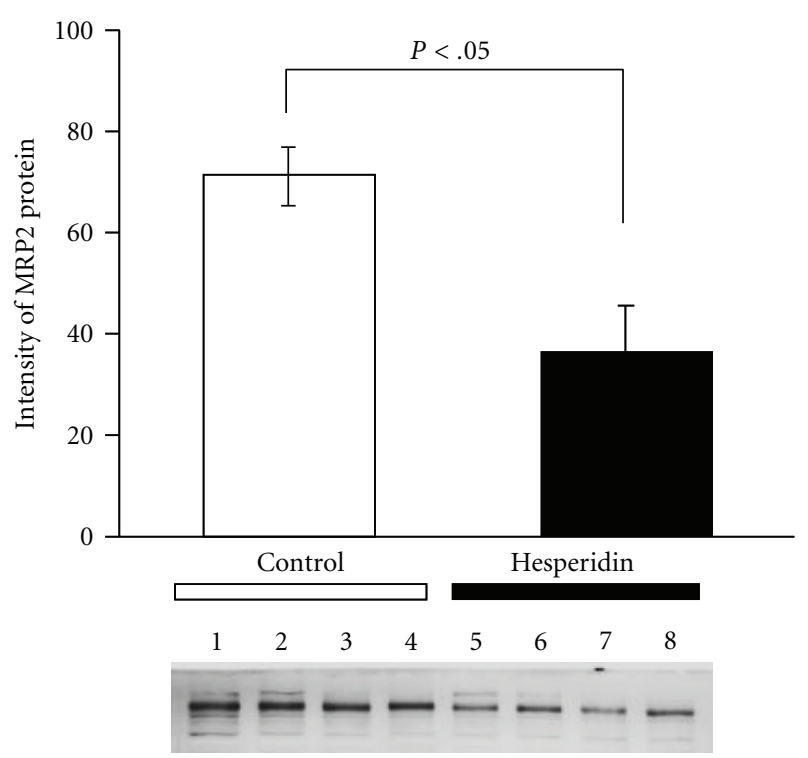

(a) Liver

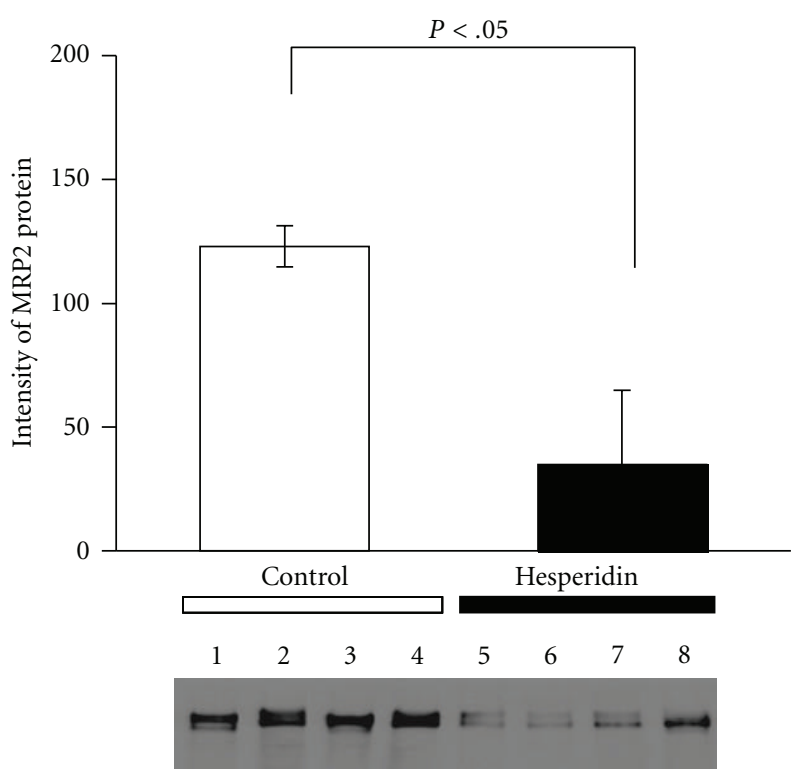

(b) Small intestine

FIGURE 5: Effect of hesperidin on Mrp2 protein levels in both the liver (a) and small intestine (b) in rats. Hesperidin instead of OJ was given to 8-week-old male Sprague-Dawley rats according to the same schedule and volume as in the OJ study. Tap water was also given as a control. The hesperidin solution was prepared at a concentration of $79 \mathrm{mg}$ per $100 \mathrm{~mL}$ of tap water. The small intestine and liver were removed $1 \mathrm{~h}$ after the last ingestion of hesperidin. Western blot analysis was performed using the same methods as in the in vitro OJ study.

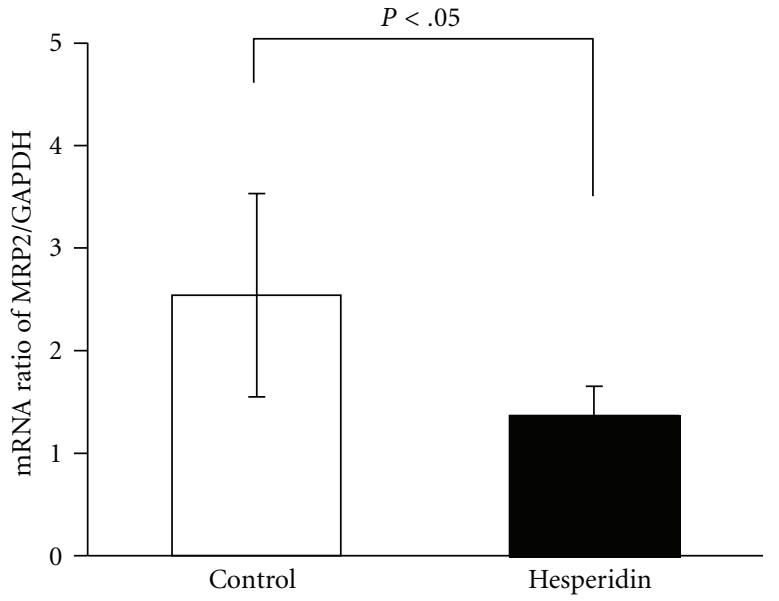

(a) Liver

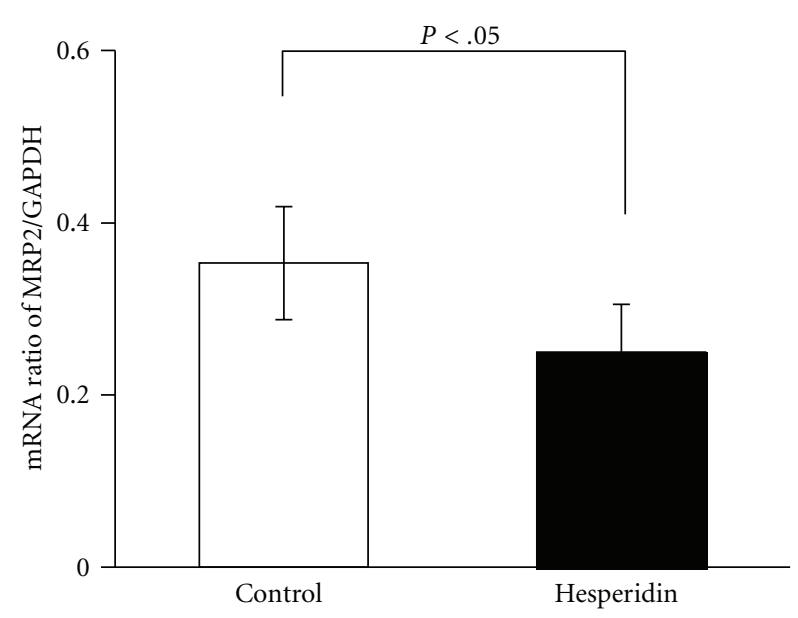

(b) Small intestine

Figure 6: Effects of hesperidin on the expression of Mrp2 mRNA in both the liver (a) and small intestine (b) in rats. Determination of Mrp2 mRNA was performed using the same methods as in the in vitro OJ study.

A similar result was obtained after the 2-day ingestion of hesperidin. Our finding that the oatp2 level increased with OJ or hesperidin intake may be also one mechanism of the increase in the PRV AUC value.

It will be necessary to examine the effects of the OJ ingestion on another transporter that participates in the transport of PRV. Further studies may also needed since oat3 in particular is believed to contribute to the active renal transport of PRV.

As the amount of hesperidin administered was preadjusted to the amount present in OJ, near quantitative evaluation may be possible. When we compared the results in the OJ and hesperidin groups, the inhibitory results were similar. Based on this similarity, we assumed that the inhibitory mechanism may be simple because most of the effect of OJ could be attributed to hesperidin.

We did not perform a confirmation study using OJ with its hesperidin content extracted. Therefore, it is possible that other factors may have contributed to the results obtained.

In addition, the possibility that other pharmacokinetic factors like metabolism or excretion may have played a role in increasing the $C_{\max }$ and AUC values cannot be ruled 
out, because we did not measure precise bioavailability. The concentration curve appears to have a slow decreasing sequence. We therefore may need to explore more detailed pharmacokinetic parameters in future studies.

In conclusion, hesperidin contained in OJ may be the substance inhibiting Mrp2.

\section{References}

[1] R. Tian, N. Koyabu, H. Takanaga, H. Matsuo, H. Ohtani, and Y. Sawada, "Effects of grapefruit juice and orange juice on the intestinal efflux of P-glycoprotein substrates," Pharmaceutical Research, vol. 19, no. 6, pp. 802-809, 2002.

[2] G. K. Dresser and D. G. Bailey, "The effects of fruit juices on drug disposition: a new model for drug interactions," European Journal of Clinical Investigation, vol. 33, no. 2, pp. 10-16, 2003.

[3] M. Haria and D. McTavish, "Pravastatin: a reappraisal of its pharmacological properties and clinical effectiveness in the management of coronary heart disease," Drugs, vol. 53, no. 2, pp. 299-336, 1997.

[4] Y. U. Koitabashi, T. Kumai, N. Matsumoto et al., "Orange juice increased the bioavailability of pravastatin, 3-hydroxy3-methylglutaryl CoA reductase inhibitor, in rats and healthy human subjects," Life Sciences, vol. 78, no. 24, pp. 2852-2859, 2006.

[5] K. T. Kivistö, O. Grisk, U. Hofmann et al., "Disposition of oral and intravenous pravastatin in MRP2-Deficient TR rats," Drug Metabolism and Disposition, vol. 33, no. 11, pp. 1593-1596, 2005.

[6] M. Hirano, K. Maeda, S. Matsushima, Y. Nozaki, H. Kusuhara, and Y. Sugiyama, "Involvement of BCRP (ABCG2) in the biliary excretion of pitavastatin," Molecular Pharmacology, vol. 68, no. 3, pp. 800-807, 2005.

[7] J. J. Lilja, K. T. Kivistö, and P. J. Neuvonen, "Grapefruit juice increases serum concentrations of atorvastatin and has no effect on pravastatin," Clinical Pharmacology and Therapeutics, vol. 66, no. 2, pp. 118-127, 1999.

[8] B. Ameer, R. A. Weintraub, J. V. Johnson, R. A. Yost, and R. L. Rouseff, "Flavanone absorption after naringin, hesperidin, and citrus administration," Clinical Pharmacology and Therapeutics, vol. 60, no. 1, pp. 34-40, 1996.

[9] I. Erlund, E. Meririnne, G. Alfthan, and A. Aro, "Human nutrition and metabolism: plasma kinetics and urinary excretion of the flavanones naringenin and hesperetin in humans after ingestion of orange juice and grapefruit juice," Journal of Nutrition, vol. 131, no. 2, pp. 235-241, 2001.

[10] K. Otter and C. Mignat, "Determination of pravastatin in human plasma by high-performance liquid chromatography with ultraviolet detection," Journal of Chromatography B: Biomedical Applications, vol. 708, no. 1-2, pp. 235-241, 1998.

[11] I. Iacona, M. B. Regazzi, I. Buggia et al., "High-performance liquid chromatography determination of pravastatin in plasma," Therapeutic Drug Monitoring, vol. 16, no. 2, pp. 191195, 1994.

[12] S. Tatsunami, A. Ito, K. Kawata, R. Kuwabara, and K. Yamada, "Determination of pharmacokinetic parameters of stavudine in Japanese patients infected with HIV-1, using a Gaussianlike input rate function," European Journal of Drug Metabolism and Pharmacokinetics, vol. 26, no. 1-2, pp. 137-140, 2001.

[13] L. Sadphati and L. Z. Benet, "Modulation of P-glycoprotein expression by cytochrome $\mathrm{P} 4503 \mathrm{~A}$ inducers in male and female rat livers," Biochemical Pharmacology, vol. 55, no. 4, pp. 387-395, 1998.

[14] Y. Kitaoka, T. Kumai, Y. Kitaoka et al., "Nuclear factor-kappa B p65 in NMDA-induced retinal neurotoxicity," Molecular Brain Research, vol. 131, no. 1-2, pp. 8-16, 2004.

[15] M. Careri, L. Elviri, A. Mangia, and M. Musci, "Spectrophotometric and coulometric detection in the high-performance liquid chromatography of flavonoids and optimization of sample treatment for the determination of quercetin in orange juice," Journal of Chromatography A, vol. 881, no. 1-2, pp. 449460, 2000.

[16] T. Tokui, D. Nakai, R. Nakagomi, H. Yawo, T. Abe, and Y. Sugiyama, "Pravastatin, an HMG-CoA reductase inhibitor, is transported by rat organic anion transporting polypeptide, oatp2," Pharmaceutical Research, vol. 16, no. 6, pp. 904-908, 1999.

[17] B. Hagenbuch and P. J. Meier, "The superfamily of organic anion transporting polypeptides," Biochimica et Biophysica Acta, vol. 1609, no. 1, pp. 1-18, 2003.

[18] M. Yamazaki, S. Akiyama, K. Ni'Inuma, R. Nishigaki, and Y. Sugiyama, "Biliary excretion of pravastatin in rats: contribution of the excretion pathway mediated by canalicular multispecific organic anion transporter (cMOAT)," Drug Metabolism and Disposition, vol. 25, no. 10, pp. 1123-1129, 1997.

[19] M. Hirano, K. Maeda, S. Matsushima, Y. Nozaki, H. Kusuhara, and Y. Sugiyama, "Involvement of BCRP (ABCG2) in the biliary excretion of pitavastatin," Molecular Pharmacology, vol. 68, no. 3, pp. 800-807, 2005.

[20] W. Jacobsen, G. Kirchner, K. Hallensleben et al., "Comparison of cytochrome P-450-dependent metabolism and drug interactions of the 3-hydroxy-3-methylglutaryl-CoA reductase inhibitors lovastatin and pravastatin in the liver," Drug Metabolism and Disposition, vol. 27, no. 2, pp. 173-179, 1999.

[21] Y. Gotoh, H. Suzuki, S. Kinoshita, T. Hirohashi, Y. Kato, and Y. Sugiyama, "Involvement of an organic anion transporter (canalicular multispecific organic anion transporter/multidrug resistance-associated protein 2) in gastrointestinal secretion of glutathione conjugates in rats," Journal of Pharmacology and Experimental Therapeutics, vol. 292, no. 1, pp. 433-439, 2000.

[22] R. Masereeuw, S. Notenboom, P. H. E. Smeets, A. C. Wouterse, and F. G. M. Russel, "Impaired renal secretion of substrates for the multidrug resistance protein 2 in mutant transportdeficient (TR) rats," Journal of the American Society of Nephrology, vol. 14, no. 11, pp. 2741-2749, 2003.

[23] K. Ito, H. Suzuki, T. Hirohashi, K. Kume, T. Shimizu, and Y. Sugiyama, "Molecular cloning of canalicular multispecific organic anion transporter defective in EHBR," American Journal of Physiology, vol. 272, no. 1, pp. G16-G22, 1997.

[24] P. Borst, R. Evers, M. Kool, and J. Wijnholds, "A family of drug transporters: the multidrug resistance-associated proteins," Journal of the National Cancer Institute, vol. 92, no. 16, pp. 1295-1302, 2000.

[25] T. Komai, K. Kawai, T. Tokui et al., "Disposition and metabolism of pravastatin sodium in rats, dogs and monkeys," European Journal of Drug Metabolism and Pharmacokinetics, vol. 17, no. 2, pp. 103-113, 1992.

[26] T. Abe, M. Kakyo, H. Sakagami et al., "Molecular characterization and tissue distribution of a new organic anion transporter subtype (oatp3) that transports thyroid hormones and taurocholate and comparison with oatp2," The Journal of Biological Chemistry, vol. 273, no. 35, pp. 22395-22401, 1998. 
[27] B. Hagenbuch and P. J. Meier, "The superfamily of organic anion transporting polypeptides," Biochimica et Biophysica Acta, vol. 1609, no. 1, pp. 1-18, 2003.

[28] B. Hsiang, Y. Zhu, Z. Wang et al., "A novel human hepatic organic anion transporting polypeptide (OATP2). Identification of a liver-specific human organic anion transporting polypeptide and identification of rat and human hydroxymethylglutaryl-CoA reductase inhibitor transporters," The Journal of Biological Chemistry, vol. 274, no. 52, pp. 37161-37168, 1999. 

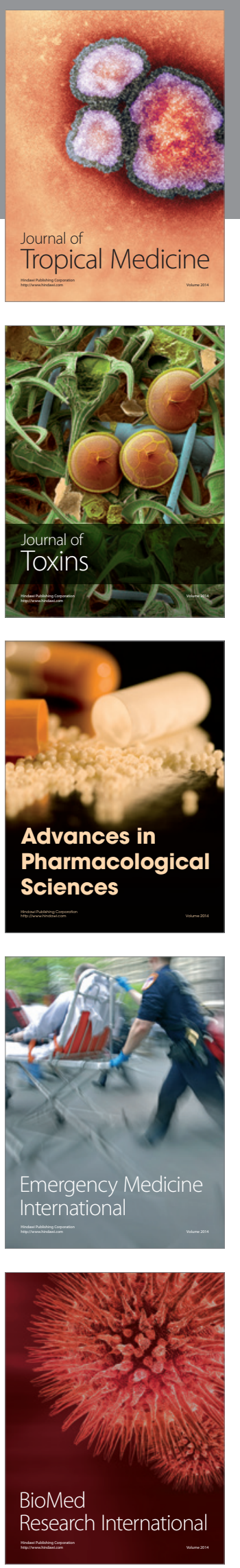
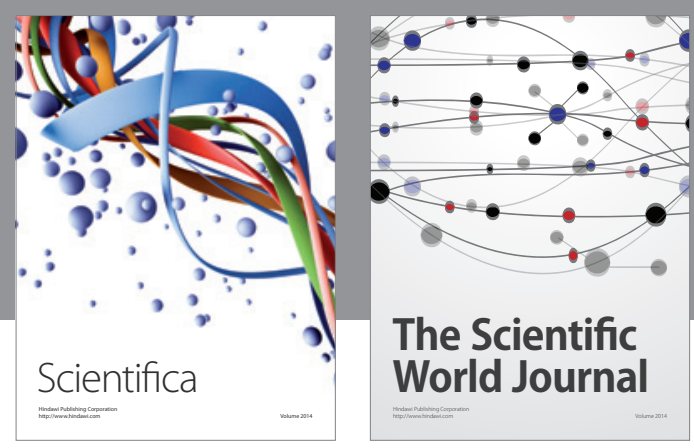

The Scientific World Journal
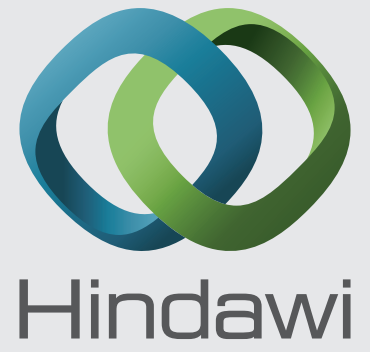

Submit your manuscripts at

http://www.hindawi.com
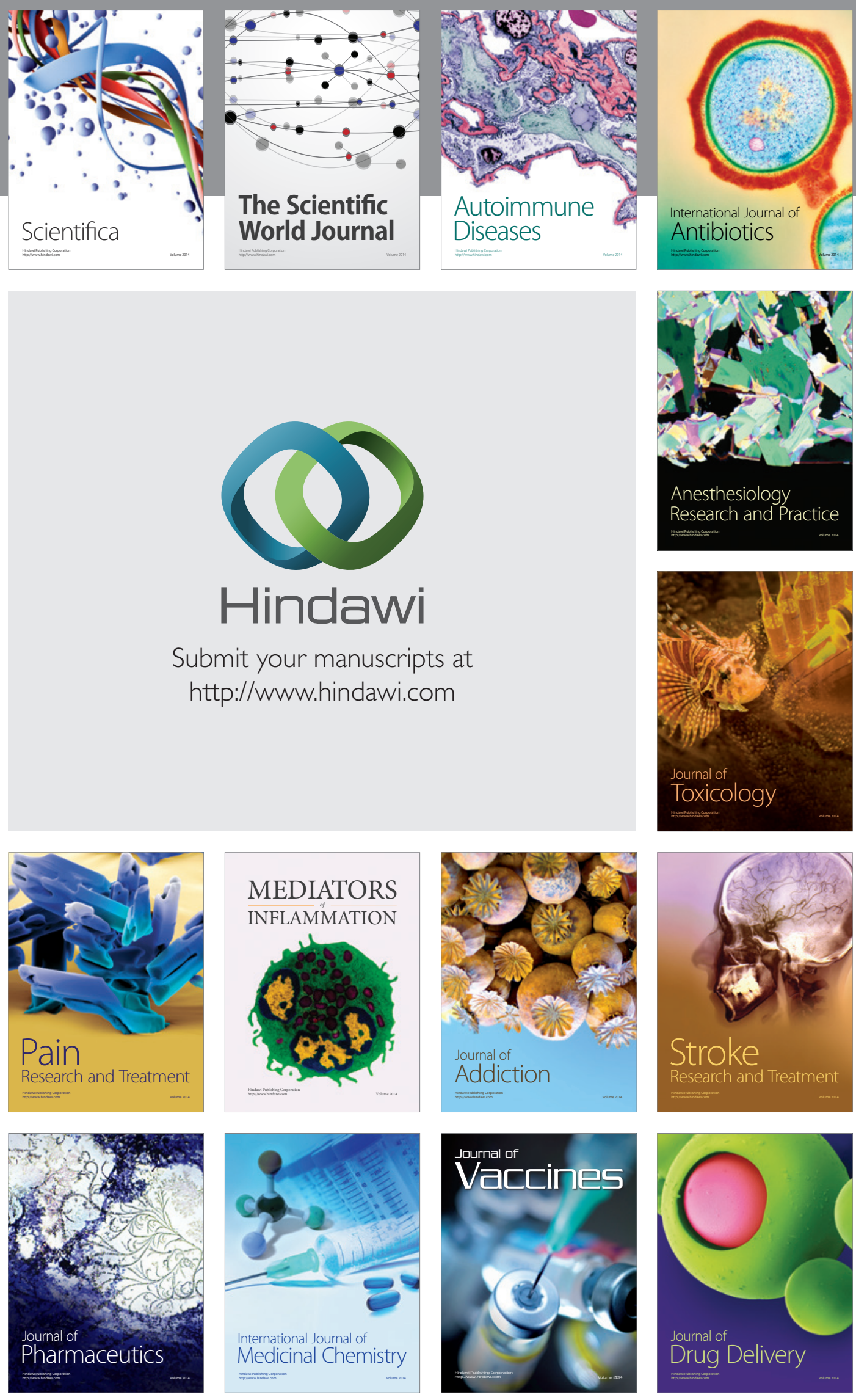